\title{
Fast computation of elastic and hydrodynamic potentials using approximate approximations
}

\author{
Flavia Lanzara ${ }^{1}(1) \cdot$ Vladimir Maz'ya $^{2,3} \cdot$ Gunther Schmidt $^{4}$ \\ Received: 18 June 2020 / Revised: 18 June 2020 / Accepted: 7 October 2020 / \\ Published online: 10 November 2020 \\ (c) The Author(s) 2020
}

\begin{abstract}
We propose fast cubature formulas for the elastic and hydrodynamic potentials based on the approximate approximation of the densities with Gaussian and related functions. For densities with separated representation, we derive a tensor product representation of the integral operator which admits efficient cubature procedures. We obtain high order approximations up to a small saturation error, which is negligible in computations. Results of numerical experiments which show approximation order $\mathscr{O}\left(h^{2 M}\right)$, $M=1,2,3,4$, are provided.
\end{abstract}

Keywords Lamé system · Linear elasticity · Stokes system · High order approximations

Mathematics Subject Classification $65 \mathrm{D} 32 \cdot 41 \mathrm{~A} 30 \cdot 41 \mathrm{~A} 63$

\section{Introduction}

In this paper we consider volume potentials which arise in the solution of the threedimensional problems in elasticity and hydrodynamics.

Linear isotropic elastic problems in the space $\mathbb{R}^{3}$ are governed by the threedimensional Lamé system

$$
\mu \Delta \mathbf{u}+(\boldsymbol{\lambda}+\mu) \operatorname{grad} \operatorname{div} \mathbf{u}+\mathbf{f}=\mathbf{0},
$$

Harmonic Analysis and PDE dedicated to Vladimir Maz'ya.

$\bowtie \quad$ Flavia Lanzara

lanzara@mat.uniroma1.it; flavia.lanzara@uniroma1.it

1 Mathematics Department, Sapienza University, P.le Aldo Moro 2, 00185 Rome, Italy

2 Department of Mathematics, University of Linköping, 58183 Linköping, Sweden

3 RUDN University, 6 Miklukho-Maklaya St, Moscow, Russia 117198

4 Lichtenberger Str. 12, 10178 Berlin, Germany

Dirkhäuser 
where $\mathbf{u}=\left(u_{1}, u_{2}, u_{3}\right)$ is the displacement vector, $\mathbf{f}=\left(f_{1}, f_{2}, f_{3}\right)$ is the volume force, $\lambda$ and $\mu$ are the Lamé constants.

The hydrodynamic potentials correspond to the linearized Navier-Stokes equations, the Stokes equations,

$$
\begin{aligned}
-v \Delta \mathbf{u}+\operatorname{grad} P & =\mathbf{f}, \\
\operatorname{div} \mathbf{u} & =0 .
\end{aligned}
$$

Here $\mathbf{u}=\left(u_{1}, u_{2}, u_{3}\right)$ is the velocity vector, $\mathbf{f}=\left(f_{1}, f_{2}, f_{3}\right)$ is the volume force, $P$ denotes the pressure and $v$ is the constant viscosity coefficient. The Eqs. (1.2)-(1.3) are considered in the whole space $\mathbb{R}^{3}$.

Suppose that $f_{\ell} \in \mathscr{S}\left(\mathbb{R}^{3}\right), \ell=1,2,3$. By $\mathscr{S}\left(\mathbb{R}^{3}\right)$ we denote the Schwartz space of smooth and rapidly decaying functions. A solution of (1.1) is given by the volume potential

$$
u_{k}(\mathbf{x})=\Gamma^{(k)} \mathbf{f}(\mathbf{x})=\sum_{\ell=1}^{3} \Gamma^{(k, \ell)}\left(f_{\ell}\right)(\mathbf{x}), \quad k=1,2,3,
$$

with

$$
\Gamma^{(k, \ell)}(g)(\mathbf{x})=\int_{\mathbb{R}^{3}} \Gamma_{k \ell}(\mathbf{x}-\mathbf{y}) g(\mathbf{y}) d \mathbf{y}, \quad k, \ell=1,2,3 .
$$

Here $\left\{\Gamma_{k \ell}\right\}_{k, \ell=1,2,3}$ is the Kelvin fundamental matrix ([7, p. 84])

$$
\Gamma_{k \ell}(\mathbf{x})=\frac{\lambda^{\prime} \delta_{k \ell}}{8 \pi|\mathbf{x}|}+\frac{1}{8 \pi \mu^{\prime}} \frac{x_{k} x_{\ell}}{|\mathbf{x}|^{3}}
$$

with

$$
\lambda^{\prime}=\frac{\lambda+3 \mu}{\mu(\lambda+2 \mu)}, \quad \mu^{\prime}=\frac{\lambda+\mu}{\mu(\lambda+2 \mu)}, \quad \lambda>0, \mu>0 .
$$

The system (1.2)-(1.3) is solved by the volume potential ([8, p. 51])

$$
u_{k}(\mathbf{x})=\sum_{\ell=1}^{3} \Psi^{(k, \ell)}\left(f_{\ell}\right)(\mathbf{x}), \quad k=1,2,3
$$

where

$$
\Psi^{(k, \ell)}(g)(\mathbf{x})=\int_{\mathbb{R}^{3}} \Psi_{k \ell}(\mathbf{x}-\mathbf{y}) g(\mathbf{y}) d \mathbf{y}, \quad k, \ell=1,2,3,
$$

with the fundamental solution ([8, p. 51])

$$
\Psi_{k \ell}(\mathbf{x})=\frac{1}{8 \pi v}\left(\frac{\delta_{k \ell}}{|\mathbf{x}|}+\frac{x_{k} x_{\ell}}{|\mathbf{x}|^{3}}\right)
$$


and

$$
P(\mathbf{x})=-\sum_{\ell=1}^{3} \frac{\partial}{\partial x_{\ell}} \frac{1}{4 \pi} \int_{\mathbb{R}^{3}} \frac{f_{\ell}(\mathbf{y})}{|\mathbf{x}-\mathbf{y}|} d \mathbf{y} .
$$

The functions $\mathbf{u}=\left(u_{1}, u_{2}, u_{3}\right)$ in (1.4) and (1.6), and $P$ in (1.8) satisfy the conditions $|\mathbf{u}(\mathbf{x})| \rightarrow 0, P(\mathbf{x}) \rightarrow 0$ and $|\operatorname{grad} P(\mathbf{x})| \rightarrow 0$ as $|\mathbf{x}| \rightarrow \infty$.

In this paper we propose a fast method of an arbitrary high order for approximating the potentials (1.4), (1.6) and (1.8), which combines the method of approximate approximations (cf. [17]) with the idea of tensor structured approximation (cf. e.g. [1-6]).

The concept of approximate approximations and first related results were published by Maz'ya $[14,15]$. Various aspects of a general theory of these approximations were systematically investigated (cf. [17] and the references therein). This approximation procedure employs linear combination of smooth and rapidly decaying functions to approximate a given function with high order within a prescribed accuracy. Although in general the method does not converge in a rigorous sense, even simple quasiinterpolants approximate functions very accurately. Additionally, this approximation process has considerable advantages due to the great flexibility in the choice of approximating functions. This flexibility makes it possible to get simple formulas for the approximation of various integral and pseudo-differential operators. Example of those cubature formulas for important integral operators of mathematical physics on $\mathbb{R}^{3}$ were studied in [16]. By combining semi-analytic cubature formulas for volume potentials based on approximate approximations with the strategy of separated representations, we derive a method for approximating volume potentials, which is accurate and fast also in the multidimensional case and provides approximation formulas of high order. This approach was introduced for the first time in [9] to obtain fast cubature of high-dimensional harmonic potentials. Fast cubature formulas of advection-diffusion potentials in the frame of approximate approximations have been obtained in [10]. In [11] the procedure has been extended to parabolic problems of second order and in [12] to the biharmonic operator.

We describe the idea for the elastic potential (1.4). The hydrodynamic potential will be considered in Sect. 4. Our approximation formulas are based on replacing the density $\mathbf{f}$ of the integral operator (1.4) by quasi-interpolants of the form

$$
\mathscr{M}_{h} \mathbf{f}(\mathbf{x}):=\mathscr{D}^{-3 / 2} \sum_{\mathbf{m} \in \mathbb{Z}^{3}} \mathbf{f}(h \mathbf{m}) \eta\left(\frac{\mathbf{x}-h \mathbf{m}}{h \sqrt{\mathscr{D}}}\right)
$$

with fixed positive parameters $h$ and $\mathscr{D}$ and with some generating function $\eta$ sufficiently smooth and of rapid decay chosen such that the integrals

$$
\Gamma^{(k, \ell)}(\eta)(\mathbf{x})=\int_{\mathbb{R}^{3}} \Gamma_{k \ell}(\mathbf{x}-\mathbf{y}) \eta(\mathbf{y}) d \mathbf{y}, \quad k, \ell=1,2,3
$$


can be effectively determined. Then the sum

$$
\Gamma^{(k, \ell)}\left(\mathscr{M}_{h} f_{\ell}\right)(\mathbf{x})=\frac{h^{2}}{\sqrt{\mathscr{D}}} \sum_{\mathbf{m} \in \mathbb{Z}^{3}} f_{\ell}(h \mathbf{m}) \Gamma^{(k, \ell)}(\eta)\left(\frac{\mathbf{x}-h \mathbf{m}}{h \sqrt{\mathscr{D}}}\right)
$$

provides an approximation formula for $\Gamma^{(k, \ell)}\left(f_{\ell}\right), k, \ell=1,2,3$.

If the generating function $\eta$ satisfies the moment conditions of order $\mathrm{N}$

$$
\int_{\mathbb{R}^{3}} \eta(\mathbf{x}) d \mathbf{x}=1, \quad \int_{\mathbb{R}^{3}} \eta(\mathbf{x}) \mathbf{x}^{\alpha} d \mathbf{x}=0 \quad \forall \alpha: 1 \leq|\alpha|<N,
$$

then at any point $\mathbf{x}$

$$
\left|f_{\ell}(\mathbf{x})-\mathscr{M}_{h} f_{\ell}(\mathbf{x})\right| \leq c_{f_{\ell}, \eta}\left((h \sqrt{\mathscr{D}})^{N}+\varepsilon_{0}(\eta, \mathscr{D})\right)
$$

(cf. [17, p. 34]) . A proper choice of the parameter $\mathscr{D}$ allows to make the saturation error $\varepsilon_{0}(\eta, \mathscr{D})$ as small as necessary, e.g., less than the precision machine. The cubature error $\Gamma^{(k, \ell)}\left(f_{\ell}\right)-\Gamma^{(k, \ell)}\left(\mathscr{M}_{h} f_{\ell}\right)=\Gamma^{(k, \ell)}\left(f_{\ell}-\mathscr{M}_{h} f_{\ell}\right)$ can be estimated by using mapping properties of the integral operator and error estimates of the quasi-interpolant (1.9). Moreover, due to the smoothing properties of the integral operators $\Gamma^{(k, \ell)}$ the corresponding approximations converge with the rate $(h \sqrt{\mathscr{D}})^{N}+h^{2} \varepsilon_{0}(\eta, \mathscr{D})$ in some uniform or $L_{p}$-norm (cf. [17, p. 113]).

Analytic formulas for $\Gamma^{(k, \ell)}\left(\eta_{2 M}\right)$ with the radial generating functions

$$
\eta_{2 M}(\mathbf{x})=\pi^{-3 / 2} L_{M-1}^{(3 / 2)}\left(|\mathbf{x}|^{2}\right) \mathrm{e}^{-|\mathbf{x}|^{2}}, \quad M=1,2, \ldots
$$

were obtained in [17, pp. 108-113]. The functions (1.11) satisfy the moment conditions (1.10) of order $2 M$ and the sum $\Gamma^{(k, \ell)}\left(\mathscr{M}_{h} f_{\ell}\right)$ provides a semi-analytic cubature formula for $\Gamma^{(k, \ell)}\left(f_{\ell}\right)$ of order $\mathscr{O}\left((h \sqrt{\mathscr{D}})^{2 M}+h^{2} \mathrm{e}^{-\mathscr{D} \pi^{2}}\right)$.

The computation of $\Gamma^{(k, \ell)}\left(\mathscr{M}_{h} f_{\ell}\right)$ at the point of a uniform grid $\{h \mathbf{j}\}$, with $\mathbf{j}=$ $\left(j_{1}, j_{2}, j_{3}\right) \in \mathbb{Z}^{3}$, leads to the multi-dimensional discrete convolution

$$
\Gamma^{(k, \ell)}\left(\mathscr{M}_{h} f_{\ell}\right)(h \mathbf{j})=\frac{h^{2}}{\sqrt{\mathscr{D}}} \sum_{\mathbf{m} \in \mathbb{Z}^{3}} f_{\ell}(h \mathbf{m}) \Gamma^{(k, \ell)}\left(\eta_{2 M}\right)\left(\frac{\mathbf{j}-\mathbf{m}}{\sqrt{\mathscr{D}}}\right), k, \ell=1,2,3 .
$$

Therefore it is very efficient to precompute and store the values $\Gamma^{(k, \ell)}\left(\eta_{2 M}\right)((\mathbf{j}-\mathbf{m}) /$ $\sqrt{\mathscr{D}})$, which can be used for any gridsize $h$. However, due to the operation number proportional to $h^{-3}$, the convolution (1.12) is not practical. We propose a method which reduces the computational effort. As basis functions, we choose

$$
\eta_{2 M}(\mathbf{x})=\prod_{j=1}^{3} \tilde{\eta}_{2 M}\left(x_{j}\right) ; \quad \tilde{\eta}_{2 M}(x)=\frac{(-1)^{M-1}}{\sqrt{\pi} 2^{2 M-1}(M-1) !} \frac{H_{2 M-1}(x) \mathrm{e}^{-x^{2}}}{x},
$$


where $H_{k}$ are the Hermite polynomials

$$
H_{k}(x)=(-1)^{k} \mathrm{e}^{x^{2}}\left(\frac{d}{d x}\right)^{k} \mathrm{e}^{-x^{2}}
$$

The functions (1.13) satisfy the moment conditions (1.10) and consequently give rise to approximation formulas of order $2 M$, plus the saturation error $\mathscr{O}\left(h^{2} \mathrm{e}^{-\mathscr{D} \pi^{2}}\right)$. Moreover, the action of three-dimensional elastic and hydrodynamic potentials onto these basis functions allows one-dimensional integral representations. For the special case of Gaussian functions, which may be even orthotropic or anisotropic, this was obtained in [17, pp. 131-136]. For example,

$$
\Gamma^{(k, \ell)}\left(\mathrm{e}^{-|\cdot|^{2}}\right)(\mathbf{x})=\int_{0}^{\infty}\left(\frac{\delta_{k \ell}}{4}\left(\frac{1}{\mu}-\frac{\mu^{\prime}}{2} \frac{t}{1+t}\right)+\frac{\mu^{\prime}}{4} \frac{t}{(1+t)^{2}} x_{\ell} x_{k}\right) \frac{\mathrm{e}^{-\frac{|\mathbf{x}|^{2}}{1+t}}}{(1+t)^{3 / 2}} d t
$$

In this paper we obtain new one-dimensional integral representations for the threedimensional integrals $\Gamma^{(k, \ell)}$ and $\Psi^{(k, \ell)}$ applied to the tensor product functions (1.13). The integrand is separated, i.e., it is a product of functions depending only on one of the variables. We show how a suitable quadrature of the one-dimensional integrals combined with the approximation of a separated representation of the density leads to a separated approximation of the integral operator. Since the separated representation of the density can be approximated with high order and only one-dimensional operations are used, the resulting method is very efficient and can provide high order approximations.

The outline of the paper is as follows. In Sect. 2 we derive one-dimensional integral representations of the three-dimensional elastic potential applied to the functions (1.13). In Sect. 3, for densities with separated representation, we derive a tensor product representation of the integral operator which admits efficient one-dimensional operations. We provide numerical tests, showing that these formulas are efficient and provide approximation of order $\mathscr{O}\left(h^{2 M}\right), M=1,2,3,4$. In Sect. 4 we apply the procedure to the hydrodynamic potentials (1.6) and (1.8), and provide approximation up to order $\mathscr{O}\left(h^{8}\right)$.

\section{Elastic potential}

Rewriting (1.5) as ([7, (1.3) p. 84])

$$
\Gamma_{k \ell}(\mathbf{x})=\frac{\delta_{k \ell}}{4 \pi \mu|\mathbf{x}|}-\frac{\mu^{\prime}}{8 \pi} \frac{\partial^{2}}{\partial x_{k} \partial x_{\ell}}|\mathbf{x}|
$$

then

$$
\int_{\mathbb{R}^{3}} \Gamma_{k \ell}(\mathbf{x}-\mathbf{y}) \mathrm{e}^{-|\mathbf{y}|^{2}} d \mathbf{y}=\frac{\delta_{k \ell}}{\mu} \mathscr{L}\left(\mathrm{e}^{-|\cdot|^{2}}\right)(\mathbf{x})-\frac{\mu^{\prime}}{2} I_{k \ell}\left(\mathrm{e}^{-|\cdot|^{2}}\right)(\mathbf{x})
$$


where $\mathscr{L}$ denotes the harmonic potential

$$
\mathscr{L}(g)(\mathbf{x})=\frac{1}{4 \pi} \int_{\mathbb{R}^{3}} \frac{g(\mathbf{y})}{|\mathbf{x}-\mathbf{y}|} d \mathbf{y}
$$

and

$$
I_{k \ell}(g)(\mathbf{x})=\frac{1}{4 \pi} \frac{\partial^{2}}{\partial x_{k} \partial x_{\ell}} \int_{\mathbb{R}^{3}}|\mathbf{x}-\mathbf{y}| g(\mathbf{y}) d \mathbf{y} .
$$

A one-dimensional integral representation with separable integrand for the harmonic potential $\mathscr{L}\left(\mathrm{e}^{-|\cdot|^{2}}\right)$ is known $([9$, p. 893]),

$$
\mathscr{L}\left(\mathrm{e}^{-|\cdot|^{2}}\right)(\mathbf{x})=\frac{1}{4} \int_{0}^{\infty} \frac{\mathrm{e}^{-\frac{|\mathbf{x}|^{2}}{1+t}}}{(1+t)^{3 / 2}} d t
$$

It remains to determine a one-dimensional integral representation for $I_{k \ell}\left(\mathrm{e}^{-|\cdot|^{2}}\right)$.

Theorem 1 The potential $I_{k \ell}\left(\mathrm{e}^{-|\cdot|^{2}}\right)$ admits the following representation

$$
I_{k \ell}\left(\mathrm{e}^{-|\cdot|^{2}}\right)(\mathbf{x})=\frac{\delta_{k \ell}}{4} \int_{0}^{\infty} \frac{t \mathrm{e}^{-\frac{|\mathbf{x}|^{2}}{1+t}}}{(1+t)^{5 / 2}} d t-\frac{x_{k} x_{\ell}}{2} \int_{0}^{\infty} \frac{t \mathrm{e}^{-\frac{|\mathbf{x}|^{2}}{1+t}}}{(1+t)^{7 / 2}} d t, \quad k, \ell=1,2,3 .
$$

Proof We use the relation ([17, (5.55) p. 109])

$$
\frac{\partial}{\partial x_{\ell}} \int_{\mathbb{R}^{3}}|\mathbf{x}-\mathbf{y}| \mathrm{e}^{-|\mathbf{y}|^{2}} d \mathbf{y}=\left(x_{\ell}+\frac{1}{2} \frac{\partial}{\partial x_{\ell}}\right) \int_{\mathbb{R}^{3}} \frac{\mathrm{e}^{-|\mathbf{y}|^{2}}}{|\mathbf{x}-\mathbf{y}|} d \mathbf{y} .
$$

Keeping in mind (2.4), we get

$$
\frac{1}{\pi} \frac{\partial}{\partial x_{\ell}} \int_{\mathbb{R}^{3}}|\mathbf{x}-\mathbf{y}| \mathrm{e}^{-|\mathbf{y}|^{2}} d \mathbf{y}=\left(x_{\ell}+\frac{1}{2} \frac{\partial}{\partial x_{\ell}}\right) \int_{0}^{\infty} \frac{\mathrm{e}^{-\frac{|\mathbf{x}|^{2}}{1+t}}}{(1+t)^{3 / 2}} d t=x_{\ell} \int_{0}^{\infty} \frac{t \mathrm{e}^{-\frac{|\mathbf{x}|^{2}}{1+t}}}{(1+t)^{5 / 2}} d t .
$$

In view of (2.3), formula (2.5) follows.

Thus, from the formulas (2.1), (2.4) and (2.5) we obtain

$$
\Gamma^{(k, \ell)}\left(\eta_{2}\right)(\mathbf{x})=\frac{1}{4 \pi^{3 / 2}} \int_{0}^{\infty}\left(\delta_{k \ell}\left(\frac{1}{\mu}-\frac{\mu^{\prime}}{2} \frac{t}{1+t}\right)+\frac{t}{(1+t)^{2}} \mu^{\prime} x_{\ell} x_{k}\right) \frac{\mathrm{e}^{-\frac{|\mathbf{x}|^{2}}{1+t}}}{(1+t)^{3 / 2}} d t
$$

Let $M>1$. We are looking for a one-dimensional integral representation with separated integrand of the potential $\Gamma^{(k, \ell)}$ with the basis functions (1.13). Keeping in mind (2.1), we write 


$$
\Gamma^{(k, \ell)}\left(\eta_{2 M}\right)(\mathbf{x})=\frac{\delta_{k \ell}}{\mu} \mathscr{L}\left(\eta_{2 M}\right)(\mathbf{x})-\frac{\mu^{\prime}}{2} I_{k \ell}\left(\eta_{2 M}\right)(\mathbf{x})
$$

A one-dimensional integral representation for the harmonic potential applied to $\eta_{2 M}$ in (1.13) is known $([9$, p. 894])

$$
\mathscr{L}\left(\eta_{2 M}\right)(\mathbf{x})=\frac{1}{4} \int_{0}^{\infty} \frac{\mathrm{e}^{-\frac{|\mathbf{x}|^{2}}{1+t}}}{(1+t)^{3 / 2}} \prod_{j=1}^{3} \mathscr{Q}_{M}^{(0)}\left(x_{j}, t\right) d t
$$

where $\mathscr{Q}_{M}^{(0)}(x, t)$ is a polynomial in $x$ whose coefficients depend on $t$, defined by

$$
\mathscr{Q}_{M}^{(0)}(x, t)=\frac{1}{\sqrt{\pi}} \sum_{s=0}^{M-1} \frac{1}{(1+t)^{s}} \frac{(-1)^{s}}{4^{s} s !} H_{2 s}\left(\frac{x}{\sqrt{1+t}}\right) .
$$

Theorem 2 Let $M>1$. The integrals $I_{k \ell}$ in (2.3) applied to $\eta_{2 M}$ in (1.13) admit the following one-dimensional integral representation

$$
\begin{aligned}
I_{\ell \ell}\left(\eta_{2 M}\right)(\mathbf{x})= & \frac{1}{4} \int_{0}^{\infty} \frac{t \mathrm{e}^{-\frac{|\mathbf{x}|^{2}}{1+t}}}{(1+t)^{5 / 2}} \prod_{j=1}^{3} \mathscr{Q}_{M}^{(0)}\left(x_{j}, t\right) d t \\
& -\frac{1}{2} \int_{0}^{\infty} \frac{t \mathrm{e}^{-\frac{|\mathbf{x}|^{2}}{1+t}}}{(1+t)^{7 / 2}} \mathscr{Q}_{M}^{(2)}\left(x_{\ell}, t\right) \prod_{\substack{j=1 \\
j \neq \ell}}^{3} \mathscr{Q}_{M}^{(0)}\left(x_{j}, t\right) d t
\end{aligned}
$$

and, for $k \neq \ell$,

$$
I_{k \ell}\left(\eta_{2 M}\right)(\mathbf{x})=-\frac{1}{2} \int_{0}^{\infty} \frac{t \mathrm{e}^{-\frac{|\mathbf{x}|^{2}}{1+t}}}{(1+t)^{7 / 2}} \mathscr{Q}_{M}^{(1)}\left(x_{\ell}, t\right) \mathscr{Q}_{M}^{(1)}\left(x_{k}, t\right) \mathscr{Q}_{M}^{(0)}\left(x_{i}, t\right) d t
$$

denoting $i \in\{1,2,3\} \backslash\{k, \ell\}$. Here $\mathscr{Q}_{M}^{(0)}$ are given in (2.8),

$$
\begin{aligned}
& \mathscr{Q}_{M}^{(1)}(x, t)=x \mathscr{Q}_{M}^{(0)}(x, t)+\mathscr{P}_{M}^{(1)}(x, t) \\
& \mathscr{P}_{M}^{(1)}(x, t)=\frac{2}{\sqrt{\pi}} \sum_{s=1}^{M-1} \frac{1}{(1+t)^{s-1 / 2}} \frac{(-1)^{s-1}}{(s-1) ! 4^{s}} H_{2 s-1}\left(\frac{x}{\sqrt{1+t}}\right) \\
& \mathscr{Q}_{M}^{(2)}(x, t)=x \mathscr{Q}_{M}^{(1)}(x, t)+\mathscr{P}_{M}^{(2)}(x, t), \\
& \mathscr{P}_{M}^{(2)}(x, t)=\frac{1}{\sqrt{\pi}} \sum_{s=1}^{M-1} \frac{1}{(1+t)^{s-1}} \frac{(-1)^{s-1}}{(s-1) ! 4^{s}} H_{2 s}\left(\frac{x}{\sqrt{1+t}}\right)
\end{aligned}
$$


Proof Using the relation $([17$, p. 55])

$$
\tilde{\eta}_{2 M}(x)=A\left(\frac{d}{d x}\right) \mathrm{e}^{-x^{2}}, \quad A\left(\frac{d}{d x}\right)=\frac{1}{\sqrt{\pi}} \sum_{s=0}^{M-1} \frac{(-1)^{s}}{s ! 4^{s}} \frac{d^{2 s}}{d x^{2 s}}
$$

and integrating by parts we get

$$
\begin{aligned}
I_{k \ell}\left(\eta_{2 M}\right)(\mathbf{x}) & =\frac{1}{4 \pi} \frac{\partial^{2}}{\partial x_{k} \partial x_{\ell}} \int_{\mathbb{R}^{3}}|\mathbf{x}-\mathbf{y}| \prod_{j=1}^{3} \tilde{\eta}_{2 M}\left(y_{j}\right) d \mathbf{y} \\
& =\prod_{j=1}^{3} A\left(\frac{\partial}{\partial x_{j}}\right) I_{k \ell}\left(\mathrm{e}^{-|\cdot|^{2}}\right)(\mathbf{x}) .
\end{aligned}
$$

Therefore, by using (2.5), we derive that

$$
\begin{aligned}
I_{k \ell}\left(\eta_{2 M}\right)(\mathbf{x})= & \frac{\delta_{k \ell}}{4} \prod_{j=1}^{3} A\left(\frac{\partial}{\partial x_{j}}\right) \int_{0}^{\infty} \frac{t \mathrm{e}^{-\frac{|\mathbf{x}|^{2}}{1+t}}}{(1+t)^{5 / 2}} d t \\
& -\prod_{j=1}^{3} A\left(\frac{\partial}{\partial x_{j}}\right)\left(\frac{x_{k} x_{\ell}}{2} \int_{0}^{\infty} \frac{t \mathrm{e}^{-\frac{|\mathbf{x}|^{2}}{1+t}}}{(1+t)^{7 / 2}} d t\right) .
\end{aligned}
$$

Note that

$$
\frac{d^{\ell}}{d x^{\ell}} \mathrm{e}^{-\frac{x^{2}}{1+t}}=\frac{(-1)^{\ell} \mathrm{e}^{-\frac{x^{2}}{1+t}}}{(1+t)^{\ell / 2}} H_{\ell}\left(\frac{x}{\sqrt{1+t}}\right)
$$

Then it is easy to verify that $\mathscr{Q}_{M}^{(0)}$ satisfies the equation

$$
A\left(\frac{d}{d x}\right) \mathrm{e}^{-\frac{x^{2}}{1+t}}=\mathscr{Q}_{M}^{(0)}(x, t) \mathrm{e}^{-\frac{x^{2}}{1+t}} .
$$

Hence,

$$
\prod_{j=1}^{3} A\left(\frac{\partial}{\partial x_{j}}\right) \int_{0}^{\infty} \frac{t \mathrm{e}^{-\frac{|\mathbf{x}|^{2}}{1+t}}}{(1+t)^{5 / 2}} d t=\int_{0}^{\infty} \frac{t \mathrm{e}^{-\frac{|\mathbf{x}|^{2}}{1+t}}}{(1+t)^{5 / 2}} \prod_{j=1}^{3} \mathscr{Q}_{M}^{(0)}\left(x_{j}, t\right) d t
$$

Let $\mathscr{Q}_{M}^{(1)}(x, t)$ and $\mathscr{Q}_{M}^{(2)}(x, t)$ be polynomials in $x$ such that

$$
\begin{aligned}
& A\left(\frac{d}{d x}\right)\left(x \mathrm{e}^{-\frac{x^{2}}{1+t}}\right)=\mathscr{Q}_{M}^{(1)}(x, t) \mathrm{e}^{-\frac{x^{2}}{1+t}}, \\
& A\left(\frac{d}{d x}\right)\left(x^{2} \mathrm{e}^{-\frac{x^{2}}{1+t}}\right)=\mathscr{Q}_{M}^{(2)}(x, t) \mathrm{e}^{-\frac{x^{2}}{1+t}} .
\end{aligned}
$$


Hence, for $\ell=1,2,3$,

$$
\begin{aligned}
& \prod_{j=1}^{3} A\left(\frac{\partial}{\partial x_{j}}\right)\left(x_{\ell}^{2} \int_{0}^{\infty} \frac{t \mathrm{e}^{-\frac{|\mathbf{x}|^{2}}{1+t}}}{(1+t)^{7 / 2}} d t\right) \\
& =\int_{0}^{\infty} \frac{t}{(1+t)^{7 / 2}} A\left(\frac{\partial}{\partial x_{\ell}}\right)\left(x_{\ell}^{2} \mathrm{e}^{-x_{\ell}^{2} /(1+t)}\right) \prod_{\substack{j=1 \\
j \neq \ell}}^{3} A\left(\frac{\partial}{\partial x_{j}}\right)\left(\mathrm{e}^{-x_{j}^{2} /(1+t)}\right) d t \\
& =\int_{0}^{\infty} \frac{t \mathrm{e}^{-\frac{|\mathbf{x}|^{2}}{1+t}}}{(1+t)^{7 / 2}} \mathscr{Q}_{M}^{(2)}\left(x_{\ell}, t\right) \prod_{\substack{j=1 \\
j \neq \ell}}^{3} \mathscr{Q}_{M}^{(0)}\left(x_{j}, t\right) d t .
\end{aligned}
$$

For $k \neq \ell$, with $k, \ell=1,2,3$ we have

$$
\begin{aligned}
& \prod_{j=1}^{3} A\left(\frac{\partial}{\partial x_{j}}\right)\left(x_{k} x_{\ell} \int_{0}^{\infty} \frac{t \mathrm{e}^{-\frac{|\mathbf{x}|^{2}}{1+t}}}{(1+t)^{7 / 2}} d t\right) \\
& =\int_{0}^{\infty} \frac{t}{(1+t)^{7 / 2}} A\left(\frac{\partial}{\partial x_{k}}\right)\left(x_{k} \mathrm{e}^{-x_{k}^{2} /(1+t)}\right) A\left(\frac{\partial}{\partial x_{\ell}}\right)\left(x_{\ell} \mathrm{e}^{-x_{\ell}^{2} /(1+t)}\right) A\left(\frac{\partial}{\partial x_{i}}\right)\left(\mathrm{e}^{-x_{i}^{2} /(1+t)}\right) d t \\
& =\int_{0}^{\infty} \frac{t \mathrm{e}^{-\frac{|\mathbf{x}|^{2}}{1+t}}}{(1+t)^{7 / 2}} \mathscr{Q}_{M}^{(1)}\left(x_{k}, t\right) \mathscr{Q}_{M}^{(1)}\left(x_{\ell}, t\right) \mathscr{Q}_{M}^{(0)}\left(x_{i}, t\right) d t, \quad i \in\{1,2,3\} \backslash\{k, \ell\} .
\end{aligned}
$$

In view of (2.13), (2.14), (2.17) and (2.18), the integrals $I_{\ell \ell}\left(\eta_{2 M}\right)$ and $I_{k \ell}\left(\eta_{2 M}\right), k \neq \ell$, can be written in the form (2.9) and (2.10), respectively.

Direct computation of the left hand sides in (2.15) and (2.16) leads to (2.11) and (2.12), respectively.

Remark 1 For example, for $M=2$

$$
\begin{aligned}
& \mathscr{Q}_{2}^{(0)}(x, t)=\frac{1}{\sqrt{\pi}}\left(-\frac{x^{2}}{(t+1)^{2}}+\frac{1}{2(t+1)}+1\right), \\
& \mathscr{Q}_{2}^{(1)}(x, t)=\frac{1}{\sqrt{\pi}}\left(-\frac{x^{3}}{(t+1)^{2}}+\frac{3 x}{2(t+1)}+x\right), \\
& \mathscr{Q}_{2}^{(2)}(x, t)=\frac{1}{\sqrt{\pi}}\left(-\frac{x^{4}}{(t+1)^{2}}+\left(\frac{5}{2(t+1)}+1\right) x^{2}-\frac{1}{2}\right),
\end{aligned}
$$

and, for $M=3$

$$
\mathscr{Q}_{3}^{(0)}(x, t)=\mathscr{Q}_{2}^{(0)}(x, t)+\frac{1}{\sqrt{\pi}}\left(\frac{x^{4}}{2(t+1)^{4}}-\frac{3 x^{2}}{2(t+1)^{3}}+\frac{3}{8(t+1)^{2}}\right)
$$


$\mathscr{Q}_{3}^{(1)}(x, t)=\mathscr{Q}_{2}^{(1)}(x, t)+\frac{1}{\sqrt{\pi}}\left(\frac{x^{5}}{2(t+1)^{4}}-\frac{5 x^{3}}{2(t+1)^{3}}+\frac{15 x}{8(t+1)^{2}}\right)$,

$\mathscr{Q}_{3}^{(2)}(x, t)=\mathscr{Q}_{2}^{(2)}(x, t)+\frac{1}{\sqrt{\pi}}\left(\frac{x^{6}}{2(t+1)^{4}}-\frac{7 x^{4}}{2(t+1)^{3}}+\frac{39 x^{2}}{8(t+1)^{2}}-\frac{3}{4(t+1)}\right)$.

From (2.6), (2.7) and Theorem 2, we represent the elastic potential of $\eta_{2 M}$ as

$$
\begin{aligned}
\Gamma^{(\ell, \ell)}\left(\eta_{2 M}\right)(\mathbf{x})= & \frac{1}{4} \int_{0}^{\infty}\left(\frac{1}{\mu}-\frac{\mu^{\prime}}{2} \frac{t}{(1+t)}\right) \frac{\mathrm{e}^{-\frac{|\mathbf{x}|^{2}}{1+t}}}{(1+t)^{3 / 2}} \prod_{r=1}^{3} \mathscr{Q}_{M}^{(0)}\left(x_{r}, t\right) d t \\
& +\frac{\mu^{\prime}}{4} \int_{0}^{\infty} \frac{t \mathrm{e}^{-\frac{|\mathbf{x}|^{2}}{1+t}}}{(1+t)^{7 / 2}} \mathscr{Q}_{M}^{(2)}\left(x_{\ell}, t\right) \prod_{\substack{r=1 \\
r \neq \ell}}^{3} \mathscr{Q}_{M}^{(0)}\left(x_{r}, t\right) d t
\end{aligned}
$$

and, for $k \neq \ell$

$$
\Gamma^{(k, \ell)}\left(\eta_{2 M}\right)(\mathbf{x})=\frac{\mu^{\prime}}{4} \int_{0}^{\infty} \frac{t \mathrm{e}^{-\frac{|\mathbf{x}|^{2}}{1+t}}}{(1+t)^{7 / 2}} \mathscr{Q}_{M}^{(1)}\left(x_{\ell}, t\right) \mathscr{Q}_{M}^{(1)}\left(x_{k}, t\right) \mathscr{Q}_{M}^{(0)}\left(x_{i}, t\right) d t
$$

denoting $i \in\{1,2,3\} \backslash\{k, \ell\}$. Formulas (2.19)-(2.20) are valid also for $M=1$, by assuming

$$
\mathscr{Q}_{1}^{(0)}(x, t)=1 / \sqrt{\pi}, \mathscr{Q}_{1}^{(1)}(x, t)=x / \sqrt{\pi}, \mathscr{Q}_{1}^{(2)}(x, t)=x^{2} / \sqrt{\pi} .
$$

\section{Implementation and numerical results for the elastic potential}

In this section we consider fast computation of the elastic potential (1.4) based on (2.19) and (2.20). In view of (1.12), the quadrature of the integrals (2.19) and (2.20) with certain quadrature weights $\omega_{p}$ and nodes $\tau_{p}$ leads to the approximation formulas

$$
\begin{aligned}
\Gamma^{(\ell, \ell)}(g)(h \mathbf{j}) \approx & \Gamma_{M, h}^{(\ell, \ell)}(g)(h \mathbf{j})=\frac{h^{2}}{4 \sqrt{\mathscr{D}}} \sum_{\mathbf{m} \in \mathbb{Z}^{3}} g(h \mathbf{m}) \\
& \times \sum_{p} \omega_{p}\left[\left(\frac{1}{\mu}-\frac{\mu^{\prime}}{2} \frac{\tau_{p}}{\left(1+\tau_{p}\right)}\right) \frac{\mathrm{e}^{-\frac{|\mathbf{m}-\mathbf{j}|^{2}}{\mathscr{D}\left(1+\tau_{p}\right)}}}{\left(1+\tau_{p}\right)^{3 / 2}} \prod_{r=1}^{3} \mathscr{Q}_{M}^{(0)}\left(\frac{j_{r}-m_{r}}{\sqrt{\mathscr{D}}}, \tau_{p}\right)\right. \\
& \left.+\mu^{\prime} \frac{\tau_{p} \mathrm{e}^{-\frac{|\mathbf{m}-\mathbf{j}|^{2}}{\mathscr{D}\left(1+\tau_{p}\right)}}}{\left(1+\tau_{p}\right)^{7 / 2}} \mathscr{Q}_{M}^{(2)}\left(\frac{j_{\ell}-m_{\ell}}{\sqrt{\mathscr{D}}}, \tau_{p}\right) \prod_{\substack{r=1 \\
r \neq \ell}}^{3} \mathscr{Q}_{M}^{(0)}\left(\frac{j_{r}-m_{r}}{\sqrt{\mathscr{D}}} \tau_{p}\right)\right],
\end{aligned}
$$




$$
\begin{aligned}
\Gamma^{(k, \ell)}(g)(h \mathbf{j}) \approx & \Gamma_{M, h}^{(k, \ell)}(g)(h \mathbf{j})=\frac{h^{2}}{4 \sqrt{\mathscr{D}}} \mu^{\prime} \sum_{\mathbf{m} \in \mathbb{Z}^{3}} g(h \mathbf{m}) \\
& \times \sum_{p} \omega_{p} \frac{\tau_{p} \mathrm{e}^{-\frac{|\mathbf{m}-\mathbf{j}|^{2}}{\mathscr{D}\left(1+\tau_{p}\right)}}}{\left(1+\tau_{p}\right)^{7 / 2}} \mathscr{Q}_{M}^{(1)}\left(\frac{j \ell-m_{\ell}}{\sqrt{\mathscr{D}}}, \tau_{p}\right) \mathscr{Q}_{M}^{(1)}\left(\frac{j_{k}-m_{k}}{\sqrt{\mathscr{D}}}, \tau_{p}\right) \\
& \mathscr{Q}_{M}^{(0)}\left(\frac{j_{i}-m_{i}}{\sqrt{\mathscr{D}}} \tau_{p}\right), k \neq \ell,
\end{aligned}
$$

denoting $i \in\{1,2,3\} \backslash\{k, \ell\}$. The approximation formulas $\Gamma_{M, h}^{(k, \ell)}(g)$ are very efficient if the function $g$ has separated representation, i.e., for a given accuracy $\varepsilon$ it can be represented as the sum of products of vectors in dimension 1

$$
g(\mathbf{x})=\sum_{s=1}^{S} \prod_{r=1}^{3} g_{r}^{(s)}\left(x_{r}\right)+\mathscr{O}(\varepsilon) .
$$

Then an approximate value of the three-dimensional convolutional sum $\Gamma^{(k, \ell)}(g)(h \mathbf{j})$ can be approximated using only one-dimensional operations as follows

$$
\begin{aligned}
\Gamma^{(\ell, \ell)}(g)(h \mathbf{j}) \approx & \frac{h^{2} \mathscr{D}}{4} \sum_{s=1}^{S} \sum_{p} \omega_{p}\left[\left(\frac{1}{\mu}-\frac{\mu^{\prime}}{2} \frac{\tau_{p}}{\left(1+\tau_{p}\right)}\right) \prod_{r=1}^{3} S_{r}^{(s)}\left(j_{r}, \tau_{p}\right)\right. \\
& \left.+\frac{\mu^{\prime} \tau_{p}}{1+\tau_{p}} T_{\ell}^{(s)}\left(j_{\ell}, \tau_{p}\right) \prod_{\substack{r=1 \\
r \neq \ell}}^{3} S_{r}^{(s)}\left(j_{i}, \tau_{p}\right)\right] \\
\Gamma^{(k, \ell)}(g)(h \mathbf{j}) \approx & \frac{h^{2} \mathscr{D}}{4} \mu^{\prime} \sum_{s=1}^{S} \sum_{p} \frac{\omega_{p} \tau_{p}}{1+\tau_{p}} R_{\ell}^{(s)}\left(j \ell, \tau_{p}\right) R_{k}^{(s)}\left(j_{k}, \tau_{p}\right) \\
& \times S_{i}^{(s)}\left(j_{i}, \tau_{p}\right), k \neq \ell,
\end{aligned}
$$

with the one-dimensional convolutions

$$
\begin{aligned}
& S_{i}^{(s)}(j, \tau)=\sum_{m \in \mathbb{Z}} g_{i}^{(s)}(h m) \frac{\mathrm{e}^{-\frac{(m-j)^{2}}{\mathscr{D}(1+\tau)}}}{\sqrt{\mathscr{D}} \sqrt{1+\tau}} \mathscr{Q}_{M}^{(0)}\left(\frac{m-j}{\sqrt{\mathscr{D}}}, \tau\right), \\
& R_{i}^{(s)}(j, \tau)=\sum_{m \in \mathbb{Z}} g_{i}^{(s)}(h m) \frac{\mathrm{e}^{-\frac{(m-j)^{2}}{\mathscr{D}(1+\tau)}}}{\sqrt{\mathscr{D}} \sqrt{1+\tau}} \mathscr{Q}_{M}^{(1)}\left(\frac{m-j}{\sqrt{\mathscr{D}}}, \tau\right), \\
& T_{i}^{(s)}(j, \tau)=\sum_{m \in \mathbb{Z}} g_{i}^{(s)}(h m) \frac{\mathrm{e}^{-\frac{(m-j)^{2}}{\mathscr{D}(1+\tau)}}}{\sqrt{\mathscr{D}} \sqrt{1+\tau}} \mathscr{Q}_{M}^{(2)}\left(\frac{m-j}{\sqrt{\mathscr{D}}}, \tau\right) .
\end{aligned}
$$


Table 1 Exact and approximated values of $\Gamma^{(1,1)}\left(\mathrm{e}^{-|\cdot|^{2}}\right)(x, 0,0)$ and the relative error using $\widetilde{\Gamma}_{4,0.05}^{(1,1)}$

\begin{tabular}{llll}
\hline $\mathrm{x}$ & Exact & Approximation & Relative error \\
\hline 0 & 0.375000000000000 & 0.374999999668078 & $8.851 \mathrm{E}-10$ \\
1 & 0.302359936723529 & 0.302359936621434 & $3.376 \mathrm{E}-10$ \\
2 & 0.200705110667521 & 0.200705110639672 & $1.387 \mathrm{E}-10$ \\
3 & 0.141549578051259 & 0.141549578050612 & $4.567 \mathrm{E}-12$ \\
4 & 0.108181997386834 & 0.108181997386670 & $1.521 \mathrm{E}-12$ \\
5 & 0.087293352157067 & 0.087293352157035 & $3.650 \mathrm{E}-13$ \\
\hline
\end{tabular}

We use an efficient quadrature rule based on the classical trapezoidal rule, which is exponentially converging for rapidly decaying smooth functions on the real line. We make the substitutions

$$
t=\mathrm{e}^{\xi}, \quad \xi=\alpha\left(\sigma+\mathrm{e}^{\sigma}\right), \quad \sigma=\beta\left(u-\mathrm{e}^{-u}\right)
$$

with positive parameters $\alpha$ and $\beta$ proposed in [18]. Then, the integrals (2.19), (2.20) are transformed to integrals over $\mathbb{R}$ with integrands decaying doubly exponentially in the variable $u$. Thus the classical trapezoidal rule provides very accurate approximations of the integral for a relatively small number of nodes $\tau_{p}=\tau p$, with $\tau>0$. After the substitution we have

$$
\begin{aligned}
\Gamma^{(\ell, \ell)}(g)(h \mathbf{j}) \approx & \widetilde{\Gamma}_{M, h}^{(\ell, \ell)}(g)(h \mathbf{j}) \\
= & \frac{h^{2} \mathscr{D}}{4} \tau \sum_{s=1}^{S} \sum_{p}\left[\left(\frac{1}{\mu}-\frac{\mu^{\prime}}{2} \frac{\Phi(\tau p)}{(1+\Phi(\tau p))}\right) \Phi^{\prime}(\tau p)\right. \\
& \left.\times \prod_{r=1}^{3} S_{r}^{(s)}\left(j_{r}, \Phi(\tau p)\right)+\mu^{\prime} \frac{\Phi(\tau p) \Phi^{\prime}(\tau p)}{1+\Phi(\tau p)} T_{\ell}^{(s)}(j \ell, \Phi(\tau p)) \prod_{\substack{r=1 \\
r \neq \ell}}^{3} S_{r}^{(s)}\left(j_{i}, \Phi(\tau p)\right)\right], \\
\Gamma^{(k, \ell)}(g)(h \mathbf{j}) \approx & \widetilde{\Gamma}_{M, h}^{(k, \ell)}(g)(h \mathbf{j})=\frac{h^{2} \mathscr{D}}{4} \mu^{\prime} \tau \sum_{s=1}^{S} \sum_{p} \frac{\Phi(\tau p) \Phi^{\prime}(\tau p)}{1+\Phi(\tau p)} \\
& \times R_{\ell}^{(s)}\left(j_{\ell}, \Phi(\tau p)\right) R_{k}^{(s)}\left(j_{k}, \Phi(\tau p)\right) S_{i}^{(s)}\left(j_{i}, \Phi(\tau p)\right), k \neq \ell,
\end{aligned}
$$

with $\Phi(u)=\exp \left(\alpha \beta\left(u-\mathrm{e}^{-u}\right)+\alpha \exp \left(\beta\left(u-\mathrm{e}^{-u}\right)\right)\right)$ and $i \in\{1,2,3\} \backslash\{k, \ell\}$.

We provide results of some experiments which show the accuracy and convergence rate of the method. We compute the elastic potential of the density $\mathbf{f}(\mathbf{x})=\left(\mathrm{e}^{-|\mathbf{x}|^{2}}, 0,0\right)$ which has the exact value $\mathbf{u}=\left(\Gamma^{(1,1)} \mathrm{e}^{-|\cdot|^{2}}, \Gamma^{(2,1)} \mathrm{e}^{-|\cdot|^{2}}, \Gamma^{(3,1)} \mathrm{e}^{-|\cdot|^{2}}\right)$ with (cf. [17, (5.56), p.110]) 
Table 2 Absolute error and rate of convergence for $\Gamma^{(1,1)}\left(\mathrm{e}^{-|\cdot|^{2}}\right)(1.2,1.2,1.2)$ using $\widetilde{\Gamma}_{M, h}^{(1,1)}$

\begin{tabular}{|c|c|c|c|c|c|c|c|c|}
\hline \multirow[t]{2}{*}{$h^{-1}$} & \multicolumn{2}{|l|}{$M=1$} & \multicolumn{2}{|l|}{$M=2$} & \multicolumn{2}{|l|}{$M=3$} & \multicolumn{2}{|l|}{$M=4$} \\
\hline & Error & Rate & Error & Rate & Error & Rate & Error & Rate \\
\hline 10 & $0.105 E-03$ & & $0.932 \mathrm{E}-05$ & & $0.195 \mathrm{E}-06$ & & $0.264 \mathrm{E}-08$ & \\
\hline 20 & $0.253 \mathrm{E}-04$ & 2.05 & $0.601 \mathrm{E}-06$ & 3.95 & $0.314 \mathrm{E}-08$ & 5.95 & $0.103 E-10$ & 8.00 \\
\hline 40 & $0.626 \mathrm{E}-05$ & 2.01 & $0.379 \mathrm{E}-07$ & 3.98 & $0.495 \mathrm{E}-10$ & 5.98 & $0.402 \mathrm{E}-13$ & 8.00 \\
\hline 80 & $0.156 \mathrm{E}-05$ & 2.00 & $0.237 \mathrm{E}-08$ & 3.99 & $0.774 \mathrm{E}-12$ & 5.99 & $0.111 \mathrm{E}-15$ & 8.50 \\
\hline 160 & $0.390 \mathrm{E}-06$ & 2.00 & $0.148 \mathrm{E}-09$ & 3.99 & $0.115 \mathrm{E}-13$ & 6.07 & $0.611 \mathrm{E}-15$ & \\
\hline
\end{tabular}

Table 3 Absolute error and rate of convergence for $\Gamma^{(2,1)}\left(\mathrm{e}^{-|\cdot|^{2}}\right)(0.8,0.8,0.8)$ using $\widetilde{\Gamma}_{M, h}^{(2,1)}$

\begin{tabular}{|c|c|c|c|c|c|c|c|c|}
\hline \multirow[t]{2}{*}{$h^{-1}$} & \multicolumn{2}{|l|}{$M=1$} & \multicolumn{2}{|l|}{$M=2$} & \multicolumn{2}{|l|}{$M=3$} & \multicolumn{2}{|l|}{$M=4$} \\
\hline & Error & Rate & Error & Rate & Error & Rate & Error & Rate \\
\hline 10 & $0.574 \mathrm{E}-03$ & & $0.103 \mathrm{E}-04$ & & $0.173 \mathrm{E}-06$ & & $0.181 \mathrm{E}-08$ & \\
\hline 20 & $0.147 \mathrm{E}-03$ & 1.96 & $0.661 \mathrm{E}-06$ & 3.96 & $0.277 \mathrm{E}-08$ & 5.96 & $0.641 \mathrm{E}-11$ & 8.13 \\
\hline 40 & $0.370 \mathrm{E}-04$ & 1.99 & $0.416 \mathrm{E}-07$ & 3.99 & $0.435 \mathrm{E}-10$ & 5.99 & $0.242 \mathrm{E}-13$ & 8.04 \\
\hline 80 & $0.926 \mathrm{E}-05$ & 1.99 & $0.260 \mathrm{E}-08$ & 3.99 & $0.680 \mathrm{E}-12$ & 5.99 & $0.590 \mathrm{E}-16$ & 8.68 \\
\hline 160 & $0.232 \mathrm{E}-05$ & 1.99 & $0.163 \mathrm{E}-09$ & 3.99 & $0.106 \mathrm{E}-13$ & 5.99 & $0.729 \mathrm{E}-16$ & \\
\hline
\end{tabular}

$$
\begin{aligned}
\Gamma^{(k, 1)}\left(\mathrm{e}^{-|\cdot|^{2}}\right)(\mathbf{x})= & \frac{\sqrt{\pi}}{8} \frac{\operatorname{erf}|\mathbf{x}|}{|\mathbf{x}|}\left(\lambda^{\prime} \delta_{1 k}+\mu^{\prime} \frac{x_{1} x_{k}}{|\mathbf{x}|^{2}}\right) \\
& +\frac{\mu^{\prime}}{8|\mathbf{x}|^{2}}\left(3 \frac{x_{1} x_{k}}{|\mathbf{x}|^{2}}-\delta_{1 k}\right)\left(\mathrm{e}^{-|\mathbf{x}|^{2}}-\frac{\sqrt{\pi}}{2} \frac{\operatorname{erf}|\mathbf{x}|}{|\mathbf{x}|}\right), k=1,2,3 .
\end{aligned}
$$

In Table 1 we compare the exact values $\Gamma^{(1,1)}\left(\mathrm{e}^{-|\cdot|^{2}}\right)$ and the approximate values $\Gamma_{4,0.05}^{(1,1)}\left(\mathrm{e}^{-|\cdot|^{2}}\right)$ at some grid points $(x, 0,0) \in \mathbb{R}^{3}$. The results show the accuracy of the method. In Tables 2 and 3 we report on the absolute errors and approximate rates for the potentials $\Gamma^{(1,1)}\left(\mathrm{e}^{-|\cdot|^{2}}\right)(1.2,1.2,1.2)$ and $\Gamma^{(2,1)}\left(\mathrm{e}^{-|\cdot|^{2}}\right)(0.8,0.8,0.8)$. The approximate values are computed by the formulas $\widetilde{\Gamma}_{M, h}^{(1,1)}$ and $\widetilde{\Gamma}_{M, h}^{(2,1)}$, respectively, for $M=1,2,3,4$ and uniform grids size $h=0.1 \times 2^{-k}, k=0, \ldots, 4$. For the calculations we choose the parameters $\mu=1, \lambda=2$, the quadrature rule with $\alpha=5$ and $\beta=6$ in (3.1), $\tau=0.004$ and 250 summands in the quadrature sum. We choose $\mathscr{D}=4$ to have the saturation error comparable with the double precision rounding errors. The numerical results confirm the $h^{2 M}$ convergence of the cubature formula when $M=1,2,3,4$. For $M=4$ and small $h$ the saturation error is reached. The approximation error consists of the sum of two terms $\mathscr{O}\left((h \sqrt{\mathscr{D}})^{2 M}\right)+$ $\mathscr{O}\left(h^{2} \mathrm{e}^{-\mathscr{D} \pi^{2}}\right)$. For sufficiently large $\mathscr{D}$, the saturation error $\mathscr{O}\left(h^{2} \mathrm{e}^{-\mathscr{D} \pi^{2}}\right)$ is negligible and $\widetilde{\Gamma}_{M, h}^{(k, \ell)}$ behaves in numerical computations as a usual $2 M$-order formula. In Table 4 we report on the absolute error and the convergence rate of the elastic potential $\Gamma^{(1,1)}\left(\mathrm{e}^{-|\cdot|^{2}}\right)(0,1,0)$ using formulas $\widetilde{\Gamma}_{3, h}^{(1,1)}$ and $\widetilde{\Gamma}_{4, h}^{(1,1)}$ for different values of $h$ and $\mathscr{D}$. 
Table 4 Absolute error and rate of convergence for $\Gamma^{(1,1)}\left(\mathrm{e}^{-|\cdot|^{2}}\right)(0,1,0)$ using $\widetilde{\Gamma}_{6, h}^{(1,1)}$ and $\widetilde{\Gamma}_{8, h}^{(1,1)}$ for different values of $h$ and $\mathscr{D}$

\begin{tabular}{|c|c|c|c|c|c|c|c|c|}
\hline \multirow[t]{2}{*}{$h^{-1}$} & \multicolumn{4}{|l|}{$M=3$} & \multicolumn{4}{|l|}{$M=4$} \\
\hline & $\mathscr{D}=1$ & Rate & $\mathscr{D}=2$ & Rate & $\mathscr{D}=2$ & Rate & $\mathscr{D}=3$ & Rate \\
\hline 5 & $0.534 \mathrm{E}-05$ & & $0.155 \mathrm{E}-06$ & & $0.287 \mathrm{E}-08$ & & $0.158 \mathrm{E}-07$ & \\
\hline 10 & $0.131 \mathrm{E}-05$ & 2.03 & $0.223 \mathrm{E}-08$ & 6.12 & $0.195 E-09$ & 3.88 & $0.362 \mathrm{E}-09$ & 5.44 \\
\hline 20 & $0.325 \mathrm{E}-06$ & 2.00 & $0.886 \mathrm{E}-10$ & 4.65 & $0.767 \mathrm{E}-10$ & 1.34 & $0.198 \mathrm{E}-11$ & 7.51 \\
\hline 40 & $0.810 \mathrm{E}-07$ & 2.00 & $0.156 \mathrm{E}-10$ & 2.50 & $0.192 \mathrm{E}-10$ & 1.99 & $0.838 \mathrm{E}-14$ & 7.88 \\
\hline 80 & $0.203 \mathrm{E}-07$ & 2.00 & $0.379 \mathrm{E}-11$ & 2.03 & $0.479 \mathrm{E}-11$ & 2.00 & $0.344 \mathrm{E}-14$ & 1.28 \\
\hline 160 & $0.506 \mathrm{E}-08$ & 2.00 & $0.944 \mathrm{E}-12$ & 2.00 & $0.120 \mathrm{E}-11$ & 2.00 & $0.555 \mathrm{E}-15$ & 2.63 \\
\hline
\end{tabular}

Table 5 Absolute error and rate of convergence for $\sum_{\ell=1}^{3} \Gamma^{(1, \ell)} f_{\ell}(1,0,0)$ using $\sum_{\ell=1}^{3} \widetilde{\Gamma}_{M, h}^{(1, \ell)} f_{\ell}(1,0,0)$

\begin{tabular}{|c|c|c|c|c|c|c|c|c|}
\hline \multirow[t]{2}{*}{$h^{-1}$} & \multicolumn{2}{|l|}{$M=1$} & \multicolumn{2}{|l|}{$M=2$} & \multicolumn{2}{|l|}{$M=3$} & \multicolumn{2}{|l|}{$M=4$} \\
\hline & Error & Rate & Error & Rate & Error & Rate & Error & Rate \\
\hline 10 & $0.371 \mathrm{E}-02$ & & $0.339 \mathrm{E}-04$ & & $0.137 \mathrm{E}-05$ & & $0.237 \mathrm{E}-06$ & \\
\hline 20 & $0.922 \mathrm{E}-03$ & 2.00 & $0.225 \mathrm{E}-05$ & 3.91 & $0.253 \mathrm{E}-07$ & 5.75 & $0.116 \mathrm{E}-08$ & 7.67 \\
\hline 40 & $0.230 \mathrm{E}-03$ & 2.00 & $0.143 \mathrm{E}-06$ & 3.97 & $0.413 \mathrm{E}-09$ & 5.93 & $0.480 \mathrm{E}-11$ & 7.91 \\
\hline 80 & $0.575 \mathrm{E}-04$ & 2.00 & $0.897 \mathrm{E}-08$ & 3.99 & $0.619 \mathrm{E}-11$ & 5.99 & $0.182 \mathrm{E}-13$ & 8.04 \\
\hline 160 & $0.144 \mathrm{E}-04$ & 2.00 & $0.561 \mathrm{E}-09$ & 3.99 & $0.966 \mathrm{E}-13$ & 6.00 & $0.333 \mathrm{E}-15$ & 5.77 \\
\hline
\end{tabular}

In the cubature formula based on $\eta_{6}$, if $\mathscr{D}=1$ the results indicate approximations of order 2 caused by the relatively large saturation error $\mathscr{O}\left(h^{2} \mathrm{e}^{-\mathscr{D} \pi^{2}}\right)$ with respect to the term $\mathscr{O}\left((h \sqrt{\mathscr{D}})^{2 M}\right)$. If $\mathscr{D}=2$ we get approximations of order 6 only for a relatively large value of $h$. The cubature formula based on $\eta_{8}$ gives, for $\mathscr{D}=2$, approximations of order 4 for a large value of $h$ and of order 2 for small $h$, caused by the relatively large term $\mathscr{O}\left(h^{2} \mathrm{e}^{-\mathscr{D} \pi^{2}}\right)$. If $\mathscr{D}=3$, it gives approximations of order 8 only for relatively large values of $h$ that is when the saturation error is negligible compared to the term $\mathscr{O}\left((h \sqrt{\mathscr{D}})^{2 M}\right)$.

As a second experiment, we compute the elastic potential of the density $\mathbf{f}=$ $\left(f_{1}, f_{2}, f_{3}\right)$ with components

$$
f_{1}=\mathrm{e}^{-|\mathbf{x}|^{2}}\left(\lambda+4 \mu-2(\lambda+\mu) x_{1}^{2}-2 \mu|\mathbf{x}|^{2}\right), f_{k}=-2(\lambda+\mu) \mathrm{e}^{-|\mathbf{x}|^{2}} x_{1} x_{k}, \quad k=2,3
$$

which provides the exact solution $\mathbf{u}=\left(\mathrm{e}^{-|\mathbf{x}|^{2}} / 2,0,0\right)$. In Table 5 we report on the absolute error and the approximation rate for $u_{1}=\sum_{\ell=1}^{3} \Gamma^{(1, \ell)} f_{\ell}$. The approximation values are computed by the formula $\sum_{\ell=1}^{3} \widetilde{\Gamma}_{M, h}^{(1, \ell)} f_{\ell}$ for $M=1,2,3,4, h=0.1 \times 2^{-k}$, $k=0, \ldots, 4$ and the parameter $\mathscr{D}=4$. For the calculations we assumed $\mu=\lambda=2$, $\alpha=5$ and $\beta=6$ in (3.1), the quadrature step $\tau=0.004$ and 250 summands in the quadrature sum. The numerical results confirm the $h^{2}, h^{4}, h^{6}$ and $h^{8}$ convergence of the cubature formulas when $M=1,2,3,4$, respectively. 


\section{Hydrodynamic potential}

We use representations (2.7), (2.9) and (2.10) to get one-dimensional integral representations with separated integrands for the solution of the linearized Navier-Stokes equations (1.2)-(1.3).

Let $M \geq 1$. We replace $\mathbf{f}=\left(f_{1}, f_{2}, f_{3}\right)$ in (1.6) with the quasi-interpolant (1.9) and the generating functions (1.13). Then an approximations of $\Psi^{(k, \ell)}\left(f_{\ell}\right)(\mathbf{x})$ is provided by

$$
\Psi_{M, h}^{(k, \ell)}\left(f_{\ell}\right)(\mathbf{x}):=\Psi^{(k, \ell)}\left(\mathscr{M}_{h} f_{\ell}\right)(\mathbf{x})=\frac{h^{2}}{\sqrt{\mathscr{D}}} \sum_{\mathbf{m} \in \mathbb{Z}^{3}} f_{\ell}(h \mathbf{m}) \Psi^{(k, \ell)}\left(\eta_{2 M}\right)\left(\frac{\mathbf{x}-h \mathbf{m}}{h \sqrt{\mathscr{D}}}\right)
$$

where rewriting (1.7) as

$$
\Psi_{k \ell}(\mathbf{x})=\frac{1}{v}\left(\frac{\delta_{k \ell}}{4 \pi|\mathbf{x}|}-\frac{\partial^{2}}{\partial x_{k} \partial x_{\ell}} \frac{|\mathbf{x}|}{8 \pi}\right)
$$

and combining it with (2.2) and (2.3), we have

$$
\Psi^{(k, \ell)}\left(\eta_{2 M}\right)(\mathbf{x})=\frac{\delta_{k \ell}}{v} \mathscr{L}\left(\eta_{2 M}\right)(\mathbf{x})-\frac{1}{2 v} I_{k \ell}\left(\eta_{2 M}\right)(\mathbf{x})
$$

In view of (2.7), (2.9) and (2.10), we can write $\Psi^{(k, \ell)}\left(\eta_{2 M}\right)$ in the form

$$
\begin{aligned}
\Psi^{(\ell, \ell)}\left(\eta_{2 M}\right)(\mathbf{x})= & \frac{1}{8 v} \int_{0}^{\infty} \frac{(t+2) \mathrm{e}^{-\frac{|\mathbf{x}|^{2}}{1+t}}}{(1+t)^{5 / 2}} \prod_{j=1}^{3} \mathscr{Q}_{M}^{(0)}\left(x_{j}, t\right) d t \\
& +\frac{1}{4 v} \int_{0}^{\infty} \frac{t \mathrm{e}^{-\frac{|\mathbf{x}|^{2}}{1+t}}}{(1+t)^{7 / 2}} \mathscr{Q}_{M}^{(2)}\left(x_{\ell}, t\right) \prod_{\substack{j=1 \\
j \neq \ell}}^{3} \mathscr{Q}_{M}^{(0)}\left(x_{j}, t\right) d t \\
\Psi^{(k, \ell)}\left(\eta_{2 M}\right)(\mathbf{x})= & \frac{1}{4 v} \int_{0}^{\infty} \frac{t \mathrm{e}^{-\frac{|\mathbf{x}|^{2}}{1+t}}}{(1+t)^{7 / 2}} \mathscr{Q}_{M}^{(1)}\left(x_{\ell}, t\right) \mathscr{Q}_{M}^{(1)}\left(x_{k}, t\right) \mathscr{Q}_{M}^{(0)}\left(x_{i}, t\right) d t, k \neq \ell
\end{aligned}
$$

with $i \in\{1,2,3\} \backslash\{k, \ell\}$. Analogously to Sects. 2 and 3, formulas (4.1), (4.2) can be used to construct high order cubature formulas for the $\mathbf{u}=\left(u_{1}, u_{2}, u_{3}\right)$ in (1.6). The resulting approximation formulas are very efficient if $f_{\ell}, \ell=1,2,3$, have separated representations.

Concerning the approximation of $P$ in (1.8), for (2.2) we have

$$
P(\mathbf{x})=-\sum_{\ell=1}^{3} \frac{\partial}{\partial x_{\ell}} \mathscr{L}\left(f_{\ell}\right)(\mathbf{x})
$$


An interesting feature of the cubature formulas based on the approximate approximation of the density is that the gradient of $\mathscr{L} f_{\ell}$ is approximated by the gradient of $\mathscr{L}\left(\mathscr{M}_{h} f_{\ell}\right)$, that is

$$
\frac{\partial}{\partial x_{\ell}} \mathscr{L}\left(f_{\ell}\right)(\mathbf{x}) \approx \frac{\partial}{\partial x_{\ell}} \mathscr{L}\left(\mathscr{M}_{h} f_{\ell}\right)(\mathbf{x})=\frac{h}{\mathscr{D}} \sum_{\mathbf{m} \in \mathbb{Z}^{3}} f_{\ell}(h \mathbf{m}) \frac{\partial}{\partial x_{\ell}} \mathscr{L}\left(\eta_{2 M}\right)\left(\frac{\mathbf{x}-h \mathbf{m}}{h \sqrt{\mathscr{D}}}\right)
$$

Moreover, by the smoothing properties of the harmonic potential and its gradient, the corresponding saturation errors converge with the rate $h^{2}$ and $h$, respectively, as $h$ tends to 0 (cf. [17, Theorems 4.10-4.11]).

The combination of (4.3) with (4.4) gives

$$
P(\mathbf{x}) \approx P_{M, h}(\mathbf{x})=-\frac{h}{\mathscr{D}} \sum_{\ell=1}^{3} \sum_{\mathbf{m} \in \mathbb{Z}^{3}} f_{\ell}(h \mathbf{m}) \frac{\partial}{\partial x_{\ell}} \mathscr{L}\left(\eta_{2 M}\right)\left(\frac{\mathbf{x}-h \mathbf{m}}{h \sqrt{\mathscr{D}}}\right)
$$

Theorem 3 [13, Theorem 3.2] The function $\frac{\partial}{\partial x_{\ell}} \mathscr{L}\left(\eta_{2 M}\right)(\mathbf{x})$ with $\eta_{2 M}$ in (1.13) admits the following one-dimensional integral representation

$$
\begin{aligned}
\frac{\partial}{\partial x_{\ell}} \mathscr{L}\left(\eta_{2 M}\right)(\mathbf{x})= & \frac{1}{4} \int_{0}^{\infty} \frac{\mathrm{e}^{-\frac{|\mathbf{x}|^{2}}{1+t}}}{(1+t)^{3 / 2}} \mathscr{R}_{M}\left(x_{\ell}, t\right) \prod_{\substack{r=1 \\
r \neq \ell}}^{3} \mathscr{Q}_{M}^{(0)}\left(x_{r}, t\right) d t \\
& -\frac{x_{\ell}}{2} \int_{0}^{\infty} \frac{\mathrm{e}^{-\frac{|\mathbf{x}|^{2}}{1+t}}}{(1+t)^{5 / 2}} \prod_{r=1}^{3} \mathscr{Q}_{M}^{(0)}\left(x_{r}, t\right) d t
\end{aligned}
$$

with $\mathscr{Q}_{M}^{(0)}$ given in (2.8), $\mathscr{R}_{1}(x, t)=0$ and, for $M>1$,

$\mathscr{R}_{M}(x, t)=\partial_{x} \mathscr{Q}_{M}^{(0)}(x, t)=\frac{1}{\sqrt{\pi}} \sum_{k=1}^{M-1} \frac{1}{(1+t)^{k+1 / 2}} \frac{(-1)^{k}}{4^{k-1}(k-1) !} H_{2 k-1}\left(\frac{x}{\sqrt{1+t}}\right)$.

Hence,

$$
\begin{aligned}
P_{M, h}(\mathbf{x})= & -\frac{h}{4 \mathscr{D}} \sum_{\ell=1}^{3} \sum_{\mathbf{m} \in \mathbb{Z}^{3}} f_{\ell}(h \mathbf{m}) \int_{0}^{\infty} \frac{\mathrm{e}^{-\frac{|\mathbf{x}-h \mathbf{m}|^{2}}{h^{2} \mathscr{D}(1+t)}}}{(1+t)^{3 / 2}}\left(\mathscr{R}_{M}\left(\frac{x_{\ell}-h m_{\ell}}{h \sqrt{\mathscr{D}}}, t\right) \prod_{\substack{r=1 \\
r \neq \ell}}^{3} \mathscr{Q}_{M}^{(0)}\left(\frac{x_{r}-h m_{r}}{h \sqrt{\mathscr{D}}}, t\right)\right. \\
& \left.-\frac{2}{(1+t)} \frac{x_{\ell}-h m_{\ell}}{h \sqrt{\mathscr{D}}} \prod_{r=1}^{3} \mathscr{Q}_{M}^{(0)}\left(\frac{x_{r}-h m_{r}}{h \sqrt{\mathscr{D}}}, t\right)\right) d t
\end{aligned}
$$

Based on the mapping properties of the integral operators $\Psi^{(k, \ell)}$, estimates of the cubature error can be obtained similar to those of the elastic potential. Indeed, we have $\left(\left[17\right.\right.$, p. 113]) $\Psi^{(k, \ell)}\left(f_{\ell}\right)-\Psi^{(k, \ell)}\left(\mathscr{M}_{h} f_{\ell}\right)=\mathscr{O}\left((h \sqrt{\mathscr{D}})^{2 M}+h^{2} \mathrm{e}^{-\pi^{2} \mathscr{D}}\right)$ and, from 
Table 6 Exact and approximated values of $\sum_{\ell=1}^{3} \Psi^{(1, \ell)}\left(f_{\ell}\right)(0, x, 0)$ and the relative error using $\sum_{\ell=1}^{3} \Psi_{4,0.0125}^{(1, \ell)}\left(f_{\ell}\right)$

\begin{tabular}{llll}
\hline $\mathrm{x}$ & Exact & Approximation & Relative error \\
\hline 0.4 & 0.340857515586485 & 0.340857515586378 & $3.120 \mathrm{E}-13$ \\
0.8 & 0.4218339392344439 & 0.4218339392344424 & $3.435 \mathrm{E}-14$ \\
1.2 & 0.284313310418546 & 0.284313310418561 & $5.311 \mathrm{E}-14$ \\
1.6 & 0.123687584709280 & 0.123687584709257 & $1.823 \mathrm{E}-13$ \\
2.0 & 0.036631277777468 & 0.0366312777777455 & $3.618 \mathrm{E}-13$ \\
\hline
\end{tabular}

Table 7 Absolute error and rate of convergence for $\sum_{\ell=1}^{3} \Psi^{(1, \ell)}\left(f_{\ell}\right)(0,0.6,0)$ using $\sum_{\ell=1}^{3} \Psi_{M . h}^{(1, \ell)}\left(f_{\ell}\right)$ for different values of $h$ and $M$

\begin{tabular}{|c|c|c|c|c|c|c|c|c|}
\hline \multirow[t]{2}{*}{$h^{-1}$} & \multicolumn{2}{|l|}{$M=1$} & \multicolumn{2}{|l|}{$M=2$} & \multicolumn{2}{|l|}{$M=3$} & \multicolumn{2}{|l|}{$M=4$} \\
\hline & Error & Rate & Error & Rate & Error & Rate & Error & Rate \\
\hline 5 & $0.115 \mathrm{E}+00$ & & $0.148 \mathrm{E}-01$ & & $0.175 \mathrm{D}-02$ & & $0.198 \mathrm{E}-03$ & \\
\hline 10 & $0.338 \mathrm{E}-01$ & 1.77 & $0.112 \mathrm{E}-02$ & 3.72 & $0.356 \mathrm{E}-04$ & 5.62 & $0.107 \mathrm{E}-05$ & 7.52 \\
\hline 20 & $0.883 \mathrm{E}-02$ & 1.94 & $0.737 \mathrm{E}-04$ & 3.93 & $0.596 \mathrm{E}-06$ & 5.90 & $0.456 \mathrm{E}-08$ & 7.88 \\
\hline 40 & $0.223 \mathrm{E}-02$ & 1.98 & $0.466 \mathrm{E}-05$ & 3.98 & $0.947 \mathrm{E}-08$ & 5.97 & $0.182 \mathrm{E}-10$ & 7.97 \\
\hline 80 & $0.559 \mathrm{E}-03$ & 1.99 & $0.292 \mathrm{E}-06$ & 3.99 & $0.149 \mathrm{E}-09$ & 5.99 & $0.708 \mathrm{E}-13$ & 8.00 \\
\hline 160 & $0.140 \mathrm{E}-03$ & 1.99 & $0.183 \mathrm{E}-07$ & 3.99 & $0.232 \mathrm{E}-11$ & 5.99 & $0.278 \mathrm{E}-15$ & 7.99 \\
\hline
\end{tabular}

the estimate (cf. [17, p. 86]) $\nabla \mathscr{L}\left(f_{\ell}\right)-\nabla \mathscr{L}\left(\mathscr{M}_{h} f_{\ell}\right)=\mathscr{O}\left((h \sqrt{\mathscr{D}})^{2 M}+h \mathrm{e}^{-\pi^{2} \mathscr{D}}\right)$, $\ell=1,2,3$, we get $P-P_{M, h}=\mathscr{O}\left((h \sqrt{\mathscr{D}})^{2 M}+h \mathrm{e}^{-\pi^{2} \mathscr{D}}\right)$.

We report on numerical results illustrating that the approximations of $\Psi^{(k, \ell)}$ by $\Psi_{M, h}^{(k, \ell)}$ are accurate and provide the predicted approximation rate $\mathscr{O}\left(h^{2 M}\right), M=$ $1,2,3,4$. We compute the solution of the Stokes system (1.2)-(1.3) with

$$
\mathbf{f}(\mathbf{x})=\left(\mathrm{e}^{-|\mathbf{x}|^{2}}\left(-x_{1}+2 v x_{2}\left(5-2|\mathbf{x}|^{2}\right)\right), \mathrm{e}^{-|\mathbf{x}|^{2}}\left(-x_{2}-2 v x_{1}\left(5-2|\mathbf{x}|^{2}\right)\right),-\mathrm{e}^{-|\mathbf{x}|^{2}} x_{3}\right)
$$

which has the exact value

$$
\mathbf{u}(\mathbf{x})=\operatorname{curl}\left(0,0,-\mathrm{e}^{-|\mathbf{x}|^{2}} / 2\right)=\left(x_{2} \mathrm{e}^{-|\mathbf{x}|^{2}},-x_{1} \mathrm{e}^{-|\mathbf{x}|^{2}}, 0\right), \quad P(\mathbf{x})=\mathrm{e}^{-|\mathbf{x}|^{2}} / 2 .
$$

The accuracy of the method is shown in Table 6, where we compare the exact values $\sum_{\ell=1}^{3} \Psi^{(1, \ell)}\left(f_{\ell}\right)$ and the approximate values $\sum_{\ell=1}^{3} \Psi_{4,0.0125}^{(1, \ell)}\left(f_{\ell}\right)$ at some grid points $(0, x, 0) \in \mathbb{R}^{3}$.

In Table 7 we report on the absolute errors and approximation rates for the value $\sum_{\ell=1}^{3} \Psi^{(1, \ell)}\left(f_{\ell}\right)(0,0.6,0)$. The approximate values are computed by the formulas $\sum_{\ell=1}^{3} \Psi_{M, h}^{(1, \ell)}\left(f_{\ell}\right)$ for $M=1,2,3,4$ and uniform grids size $h=0.1 \times 2^{-k}, k=$ $0, \ldots, 4$.

We conclude this section by showing accuracy and convergence rate of formula (4.5) for the approximation of $P$ in (4.3) (see Table 8). We assume $f_{1}=\mathrm{e}^{-|\mathbf{x}|^{2}}\left(3-2|\mathbf{x}|^{2}\right.$ ), 
Table 8 Absolute error and rate of convergence for $P(\mathbf{x})$ in $\mathbf{x}=(0.4,0.4,0)$ using $P_{M, h}$ in (4.5)

\begin{tabular}{|c|c|c|c|c|c|c|c|c|}
\hline \multirow[t]{2}{*}{$h^{-1}$} & \multicolumn{2}{|l|}{$M=1$} & \multicolumn{2}{|l|}{$M=2$} & \multicolumn{2}{|l|}{$M=3$} & \multicolumn{2}{|l|}{$M=4$} \\
\hline & Error & Rate & Error & Rate & Error & Rate & Error & Rate \\
\hline 10 & $0.239 \mathrm{E}-01$ & & $0.867 \mathrm{E}-03$ & & $0.314 \mathrm{E}-04$ & & $0.113 \mathrm{E}-05$ & \\
\hline 20 & $0.624 \mathrm{E}-02$ & 1.94 & $0.573 \mathrm{E}-04$ & 3.92 & $0.533 \mathrm{E}-06$ & 5.88 & $0.493 \mathrm{E}-08$ & 7.84 \\
\hline 40 & $0.158 \mathrm{E}-02$ & 1.98 & $0.363 \mathrm{E}-05$ & 3.98 & $0.851 \mathrm{E}-08$ & 5.97 & $0.198 \mathrm{E}-10$ & 7.96 \\
\hline 80 & $0.395 \mathrm{E}-03$ & 2.00 & $0.228 \mathrm{E}-06$ & 3.99 & $0.134 \mathrm{E}-09$ & 5.99 & $0.771 \mathrm{E}-13$ & 8.00 \\
\hline 160 & $0.989 \mathrm{E}-04$ & 2.00 & $0.143 \mathrm{E}-07$ & 4.00 & $0.209 \mathrm{E}-11$ & 6.00 & $0.167 \mathrm{E}-15$ & 8.85 \\
\hline
\end{tabular}

$f_{2}=f_{3}=0$, which provides, keeping in mind that $\mathscr{L}\left(f_{1}\right)=\mathrm{e}^{-|\mathbf{x}|^{2}} / 2$, the exact solution $P(\mathbf{x})=x_{1} \mathrm{e}^{-|\mathbf{x}|^{2}}$.

For the calculations we choose the parameter $v=2$, the quadrature rule with $\alpha=5$ and $\beta=6$ in (3.1), $\tau=0.003$ and 250 summands in the quadrature sum. We choose $\mathscr{D}=4$ to have the saturation error comparable with the double precision rounding errors. The numerical results confirm the $h^{2 M}$ convergence of the cubature formula when $M=1,2,3,4$.

Acknowledgements The second author was supported by the RUDN University Program 5-100.

Funding Open access funding provided by Università degli Studi di Roma La Sapienza within the CRUICARE Agreement.

\section{Compliance with ethical standards}

Conflict of interest The authors declare that there is no conflict of interest.

Open Access This article is licensed under a Creative Commons Attribution 4.0 International License, which permits use, sharing, adaptation, distribution and reproduction in any medium or format, as long as you give appropriate credit to the original author(s) and the source, provide a link to the Creative Commons licence, and indicate if changes were made. The images or other third party material in this article are included in the article's Creative Commons licence, unless indicated otherwise in a credit line to the material. If material is not included in the article's Creative Commons licence and your intended use is not permitted by statutory regulation or exceeds the permitted use, you will need to obtain permission directly from the copyright holder. To view a copy of this licence, visit http://creativecommons.org/licenses/by/4.0/.

\section{References}

1. Beylkin, G., Mohlenkamp, M.J.: Numerical-operator calculus in higher dimensions. Proc. Natl. Acad. Sci. U. S. A. 99, 10246-10251 (2002)

2. Beylkin, G., Mohlenkamp, M.J.: Algorithms for numerical analysis in high dimensions. SIAM J. Sci. Comput. 26, 2133-2159 (2005)

3. Beylkin, G., Cramer, R., Fann, G., Harrison, R.J.: Multiresolution separated representations of singular and weakly singular operators. Appl. Comput. Harmon. Anal. 23, 235-253 (2007)

4. Hackbusch, W.: Efficient convolution with the Newton potential in d dimensions. Numer. Math. 110, 449-489 (2008) 
5. Hackbusch, W., Khoromskij, B.N.: Tensor-product approximation to operators and functions in high dimensions. J. Complexity 23(4-6), 697-714 (2007)

6. Khoromskij, B.N.: Fast and accurate tensor approximation of multivariate convolution with linear scaling in dimension. J. Comput. Appl. Math. 234, 3122-3139 (2010)

7. Kupradze, V.D.: Three-Dimensional Problems of the Mathematical Theory of Elasticity and Thermoelasticity. North Holland Publication, Amsterdam (1979)

8. Ladyzhenskaya, O.A.: The Mathematical Theory of Viscous Incompressible Flow. Gordon and Breach Science Publishers, London (1969)

9. Lanzara, F., Maz'ya, V., Schmidt, G.: On the fast computation of high dimensional volume potentials. Math. Comput. 80, 887-904 (2011)

10. Lanzara, F., Maz'ya, V., Schmidt, G.: Fast cubature of volume potentials over rectangular domains by approximate approximations. Appl. Comput. Harmon. Anal. 36, 167-182 (2014)

11. Lanzara, F., Maz'ya, V., Schmidt, G.: Approximation of solutions to multidimensional parabolic equations by approximate approximations. Appl. Comput. Harmon. Anal. 41, 749-767 (2016)

12. Lanzara, F., Maz'ya, V., Schmidt, G.: Fast cubature of high dimensional biharmonic potential based on approximate approximations. Annali dell'Università di Ferrara 65, 277-300 (2019)

13. Lanzara, F., Maz'ya, V., Schmidt, G.: Approximation of solutions to nonstationary Stokes system. J. Math. Sci. 244, 436-450 (2020)

14. Maz'ya, V.: A new approximation method and its applications to the calculation of volume potentials. Boundary point method. In: 3. DFG-Kolloqium des DFG-Forschungsschwerpunktes Randelementmethoden (1991)

15. Maz'ya, V.: Approximate approximations. In: Whiteman, J.R. (ed.) The Mathematics of Finite Elements and Applications. Highlights 1993, pp. 77-104. Wiley, New York (1994)

16. Maz'ya, V., Schmidt, G.: "Approximate Approximations" and the cubature of potentials. Rend. Mat. Acc. Lincei 6, 161-184 (1995)

17. Maz’ya, V., Schmidt, G.: Approximate Approximations. AMS, Providence (2007)

18. Takahasi, H., Mori, M.: Doubly exponential formulas for numerical integration. Publ. RIMS Kyoto Univ. 9, 721-741 (1974)

Publisher's Note Springer Nature remains neutral with regard to jurisdictional claims in published maps and institutional affiliations. 